J. Korean Math. Soc. 50 (2013), No. 1, pp. 189-202

http://dx.doi.org/10.4134/JKMS.2013.50.1.189

\title{
HOLOMORPHIC MEAN LIPSCHITZ FUNCTIONS ON THE UNIT BALL OF $\mathbb{C}^{n}$
}

\author{
Ern Gun Kwon, Hong Rae Cho, and Hyungwoon Koo
}

Abstract. On the unit ball of $\mathbb{C}^{n}$, the space of those holomorphic functions satisfying the mean Lipschitz condition

$$
\int_{0}^{1} \omega_{p}(t, f)^{q} \frac{d t}{t^{1+\alpha q}}<\infty
$$

is characterized by integral growth conditions of the tangential derivatives as well as the radial derivatives, where $\omega_{p}(t, f)$ denotes the $L^{p}$ modulus of continuity defined in terms of the unitary transformations of $\mathbb{C}^{n}$.

\section{Introduction}

Let $B=B_{n}$ be the open unit ball of $\mathbb{C}^{n}$ and $S$ be the boundary of $B$. Let $v$ be the Lebesgue volume measure on $\mathbb{C}^{n}=\mathbb{R}^{2 n}$ and $\sigma$ be the surface area measure on $S$ normalized to be $\sigma(S)=1$. We denote by $H^{p}(B), 1 \leq p<\infty$, the Hardy space on $B$. We use the customary notation

$$
\|f\|_{H^{p}(B)}=\sup _{0<r<1} M_{p}(r, f) \quad \text { and } \quad M_{p}(r, g)=\left(\int_{S}|g(r \zeta)|^{p} d \sigma(\zeta)\right)^{1 / p}
$$

respectively for holomorphic $f$ and measurable $g$ on $B$. We will denote $B_{1}$ by $\mathbb{D}$.

Concerning the boundary smoothness of $H^{p}(\mathbb{D})$ functions, it is known that the growth rate of

$$
\omega_{p}(t, f)=\left(\sup _{|h| \leq t} \int_{-\pi}^{\pi}\left|f\left(e^{i(\theta+h)}\right)-f\left(e^{i \theta}\right)\right|^{p} d \theta\right)^{1 / p}
$$

Received February 21, 2012; Revised April 24, 2012.

2010 Mathematics Subject Classification. 32A30, 30H25.

Key words and phrases. mean Lipschitz condition, Besov space, mean modulus of continuity.

The first author was supported by (NRF-2010-0021986). The second author was supported by the National Research Foundation of Korea(NRF) grant funded by the Korea government (KRF-2009-0073976). The third author was supported by (NRF-2012000705). 
is closely related to that of $M_{p}\left(r, f^{\prime}\right)$. To be more precise, for $f \in H^{p}(\mathbb{D})$ and $1 \leq q<\infty, 0<\alpha<1$, the mean Lipschitz condition

$$
\int_{0}^{1} \omega_{p}(t, f)^{q} \frac{d t}{t^{1+\alpha q}}<\infty
$$

is equivalent to the Besov condition

$$
\int_{0}^{1} M_{p}\left(r, f^{\prime}\right)^{q}(1-r)^{(1-\alpha) q-1} d r<\infty .
$$

Hardy and Littlewood ([6], see also Chapter 5 of [4]) initiated this equivalence by considering the limiting case $q=\infty$, then followed several generalizations. We refer to [5] for general exponents and also refer to [12] for the corresponding results on the upper half space $\mathbb{R}_{+}^{n+1}$ in the context of harmonic functions.

The goal of this paper is to establish the $n$-variable version of the equivalence $(1.1) \Longleftrightarrow(1.2)$. We adapt the $L^{p}$-modulus of continuity $\omega_{p}(t, f)$ of $f$ as

$$
\omega_{p}(t, f)=\sup \left\{\left(\int_{S}|f(U \zeta)-f(\zeta)|^{p} d \sigma(\zeta)\right)^{1 / p}: U \in \mathcal{U},\|U-I\| \leq t\right\},
$$

where $\mathcal{U}$ denotes the group of all unitary transformations on $\mathbb{C}^{n}, I$ denotes the identity of $\mathcal{U}$, and $\|U-I\|:=\sup _{\zeta \in S}|U \zeta-\zeta|$. We consider the space $\Lambda_{\alpha}^{p, q}(B)$ which is defined to be the set of $f \in H^{p}(B)$ satisfying (1.1). Holomorphic Besov spaces satisfying growth condition of types (1.2) for several variables have been studied extensively in the literature, specially when $p=q([2],[3],[8])$. On the other hand, $\Lambda_{\alpha}^{p, q}(B)$ have not been studied adequately in their full nature as far as we are aware of.

We denote $\mathcal{R} f$ the radial derivative of $f$ in $B$ defined by

$$
\mathcal{R} f=\sum_{j=1}^{n} z_{j} \frac{\partial}{\partial z_{j}} f .
$$

For $1 \leq i, j \leq n$, we define the tangential derivatives, $\mathcal{T}_{i j}$ and $\overline{\mathcal{T}}_{i j}$, by

$$
\mathcal{T}_{i j}=\bar{z}_{i} \frac{\partial}{\partial z_{j}}-\bar{z}_{j} \frac{\partial}{\partial z_{i}}, \quad \overline{\mathcal{T}}_{i j}=z_{i} \frac{\partial}{\partial \bar{z}_{j}}-z_{j} \frac{\partial}{\partial \bar{z}_{i}} .
$$

Given a multi-index $\nu=\left(\nu_{1}, \ldots, \nu_{n}\right)$, we use the notation $T^{\nu}$ to mean

$$
T_{i_{1} j_{1}}^{\nu_{1}} \cdots T_{i_{n} j_{n}}^{\nu_{n}}
$$

for some choice of $i_{1}, \ldots, i_{n}$ and $j_{1}, \ldots, j_{n}$, where $T_{i j}$ is either $\mathcal{T}_{i j}$ or $\overline{\mathcal{T}}_{i j}$. We have the following characterization of $\Lambda_{\alpha}^{p, q}(B)$ as our main result.

Theorem 1.1. Let $0<\alpha<1$ and $1 \leq p, q<\infty$. Then, for $f \in H^{p}(B)$ the following are equivalent.

(i) $\int_{0}^{1} \omega_{p}(t, f)^{q} \frac{d t}{t^{1+\alpha q}}<\infty$. 
(ii) $\int_{0}^{1} M_{p}(r, \mathcal{R} f)^{q}(1-r)^{(1-\alpha) q-1} d r<\infty$.

(iii) $\sum_{|\nu|=2} \int_{0}^{1} M_{p}\left(r, T^{\nu} f\right)^{q}(1-r)^{(1-\alpha) q-1} d r<\infty$.

Moreover, if $0<\alpha<\frac{1}{2}$, then these are equivalent to

(iv) $\sum_{|\nu|=1} \int_{0}^{1} M_{p}\left(r, T^{\nu} f\right)^{q}(1-r)^{(1 / 2-\alpha) q-1} d r<\infty$.

Furthermore, all the left side quantities are equivalent up to addition by $\|f\|_{H^{p}(B)}^{q}$.

We also prove a higher order derivative version of Theorem 1.1. For the precise statements and their generalizations, see Theorem 2.1 and Theorem 4.1. Note that our theorem not only proves the equivalences of the holomorphic mean Lipschitz spaces with the appropriate holomorphic Besov spaces but also reveals the usual phenomenon that the tangential derivatives behave twice better than the radial derivative.

We start with proving the equivalences between Besov spaces defined in terms of the tangential derivatives and those defined in terms of the radial derivatives, in Section 2. We then prove Theorem 1.1 in Section 4 after preparing a priori estimates in Section 3 .

Throughout this paper, the exponents $p$ and $q$ range over $1 \leq p, q<\infty$ and $k, l$ denote positive integers. Functions denoted by $f$ is assumed to be holomorphic in $B$. For nonnegative quantities $X$ and $Y$, we write $X \lesssim Y$ if there exists an absolute constant $C>0$ such that $X \leq C Y$. Also, we write $X \approx Y$ if $X \lesssim Y \lesssim X$

Acknowledgements. The authors would like to express thanks to Professors M. Pavlović and K. Zhu for helpful comments.

\section{Equivalence between derivatives}

In this section, we establish parts of Theorem 1.1 by showing equivalences between mixed norms of the radial derivatives and those of the tangential derivatives.

For the notational convenience, we denote $L_{\beta}^{p, q}(g), \beta>0$, by

$$
L_{\beta}^{p, q}(g)=\left(\int_{0}^{1} M_{p}(r, g)^{q}(1-r)^{\beta q-1} d r\right)^{1 / q} .
$$

Theorem 2.1. Let $0<\alpha<\infty$. Then for $f \in H^{p}(B)$ the following are equivalent.

(i) $L_{k-\alpha}^{p, q}\left(\mathcal{R}^{k} f\right)<\infty$ for some $k>\alpha$.

(ii) $L_{k-\alpha}^{p, q}\left(\mathcal{R}^{k} f\right)<\infty$ for all $k>\alpha$.

(iii) $\sum_{|\nu|=k} L_{k / 2-\alpha}^{p, q}\left(T^{\nu} f\right)<\infty$ for some $k>2 \alpha$. 
(iv) $\sum_{|\nu|=k} L_{k / 2-\alpha}^{p, q}\left(T^{\nu} f\right)<\infty$ for all $k>2 \alpha$. Moreover, if $0<\alpha<\frac{1}{2}$, then these are equivalent to

(v) $\sum_{|\nu|=1} L_{1 / 2-\alpha}^{p, q}\left(T^{\nu} f\right)<\infty$.

Furthermore, all the left side quantities are equivalent up to addition by $\|f\|_{H^{p}(B)}$.

Before proving Theorem 2.1, we describe some prerequisites on comparing integral means, which might have been known to experts. For the conveniences of the readers, we include proofs for which we could not find references.

Recall the definition of the non-isotropic weight of a differential operator. We assign weight 1 to $\mathcal{R}$, while weight $1 / 2$ is given to $\mathcal{T}_{i j}$ and $\overline{\mathcal{T}}_{i j}$ each. We will consider differential operators $X$ appearing as composition

$$
X=X_{1} \cdots X_{k},
$$

where each $X_{l}$ is $\mathcal{R}$ or one of $\mathcal{T}_{i j}$ or $\overline{\mathcal{T}}_{i j}$. For such an operator, its weight is defined to be the sum of each weights of $X_{l}$.

For $z \in B$ and $\delta>0$, let $P(z, \delta)$ be the non-isotropic polydisc defined as follows. If $z=r \zeta, 0 \leq r<1, \zeta \in S$, pick $\eta_{2}, \ldots, \eta_{n}$ so that $\left\{\zeta, \eta_{2}, \ldots, \eta_{n}\right\}$ is an orthonormal basis of $\mathbb{C}^{n}$. Then

$$
P(z, \delta)=\left\{w=z+\lambda \zeta+\sum_{j=2}^{n} \lambda_{j} \eta_{j}:|\lambda|<\delta,\left|\lambda_{j}\right|<\delta^{1 / 2}, j=2, \ldots, n\right\} .
$$

For $\zeta \in S$ and $0<\delta<1$, let

$$
Q(\zeta, \delta)=\left\{\eta \in S:|1-\langle\eta, \zeta\rangle|^{1 / 2}<\delta\right\}
$$

Then, it is not hard to see that there is a constant $\epsilon>0$ such that for all $1 / 2<r<1$ and $\delta=1-r$

$$
P(r \zeta, \epsilon \delta) \subset\left\{t \eta: r-\frac{\delta}{4}<t<r+\frac{\delta}{4}, \eta \in Q(\zeta, \sqrt{\delta})\right\} .
$$

The following is a weak version of Lemma 2.5 of [1].

Lemma $2.2([1])$. Let $X$ and $Y$ be the differential operators of the form (2.2) with the weight of $X$ being $m$. Then we have

$$
|X Y f(z)|^{p} \lesssim \frac{1}{\delta^{n+1+m p}} \int_{P(z, \delta)}|Y f(w)|^{p} d v(w)
$$

for $P(z, \delta) \subset B$.

Corollary 2.3. Let $1 / 2<r<1$. Let $X$ and $Y$ be the differential operators of the form (2.2) with the weight of $X$ being $m$. Then we have

$$
M_{p}(r, X Y f) \lesssim \frac{M_{p}(\tilde{r}, Y f)}{(1-r)^{m}}, \quad \tilde{r}=r+(1-r) / 4
$$


Proof. Let $\delta=1-r$. Choose $\epsilon>0$ sufficiently small to have (2.3). Since $\sigma(Q(\eta, \sqrt{\delta})) \lesssim \delta^{n}$, by $(2.4)$ and Fubini's theorem, we have

$$
\begin{aligned}
M_{p}^{p}(r, X Y f) & \lesssim \frac{1}{\delta^{p m+n+1}} \int_{\zeta \in S} \int_{w \in P(r \zeta, \epsilon \delta)}|Y f(w)|^{p} d v(w) d \sigma(\zeta) \\
& \lesssim \frac{1}{\delta^{p m+n+1}} \int_{\zeta \in S} \int_{r-\delta / 4}^{r+\delta / 4} \int_{\eta \in Q(\zeta, \sqrt{\delta})}|Y f(t \eta)|^{p} d \sigma(\eta) d t d \sigma(\zeta) \\
& =\frac{1}{\delta^{p m+n+1}} \int_{r-\delta / 4}^{r+\delta / 4} \int_{\eta \in S} \sigma(Q(\eta, \sqrt{\delta}))|Y f(t \eta)|^{p} d \sigma(\eta) d t \\
& \lesssim \frac{1}{\delta^{p m+1}} \int_{r-\delta / 4}^{r+\delta / 4} M_{p}^{p}(t, Y f) d t .
\end{aligned}
$$

Since the radial or the tangential differentiation preserves the harmonicity of $f$, the increasing property of $M_{p}(t, Y f)$ in $t$ completes the proof.

Let $\overline{\mathcal{R}}=\sum_{j=1}^{n} \overline{z_{j}} \frac{\partial}{\partial \bar{z}_{j}}$. Then a straightforward calculation shows that $\mathcal{R} \overline{\mathcal{R}}=$ $\overline{\mathcal{R}} \mathcal{R}$ and

$$
\begin{array}{ll}
\mathcal{R} \mathcal{T}_{i j}=\mathcal{T}_{i j} \mathcal{R}-\mathcal{T}_{i j}, & \mathcal{R} \overline{\mathcal{T}}_{i j}=\overline{\mathcal{T}}_{i j} \mathcal{R}+\overline{\mathcal{T}}_{i j}, \\
\overline{\mathcal{R}} \mathcal{T}_{i j}=\mathcal{T}_{i j} \overline{\mathcal{R}}+\mathcal{T}_{i j}, & \overline{\mathcal{R}} \overline{\mathcal{T}}_{i j}=\overline{\mathcal{T}}_{i j} \overline{\mathcal{R}}-\overline{\mathcal{T}}_{i j} .
\end{array}
$$

This implies

$$
(\mathcal{R}+\overline{\mathcal{R}}) X=X(\mathcal{R}+\overline{\mathcal{R}})
$$

for the differential operator $X$ of the form (2.2).

Lemma 2.4. Let $X$ be the differential operators of the form (2.2). Then, for $1 / 2<r<1$ we have

$$
M_{p}(r, X f) \lesssim \sup _{|z|<1 / 2}|f(z)|+\int_{0}^{r}(r-t)^{k-1} M_{p}\left(t, X \mathcal{R}^{k} f\right) d t,
$$

and

$$
M_{p}\left(r, T^{\nu} f\right) \lesssim \sup _{|z|<1 / 2}|f(z)|+\sum_{|\mu|=|\nu|+k} \int_{0}^{r}(r-t)^{k / 2-1} M_{p}\left(t, T^{\mu} f\right) d t .
$$

Proof. By the fundamental theorem of calculus together with (2.5),

$$
\begin{aligned}
M_{p}(r, X f) & \lesssim \sup _{|z|<1 / 3}|X f(z)|+\left(\int_{S}|X f(r \zeta)-X f(\zeta / 4)|^{p} d \sigma(\zeta)\right)^{1 / p} \\
& \lesssim \sup _{|z|<1 / 3}|X f(z)|+\left\{\int_{S}\left(\int_{1 / 4}^{r}|(\mathcal{R}+\overline{\mathcal{R}}) X f(t \zeta)| d t\right)^{p} d \sigma(\zeta)\right\}^{1 / p} \\
& =\sup _{|z|<1 / 3}|X f(z)|+\left\{\int_{S}\left(\int_{1 / 4}^{r}|X \mathcal{R} f(t \zeta)| d t\right)^{p} d \sigma(\zeta)\right\}^{1 / p}
\end{aligned}
$$


Applying Minkowski's integral inequality to the last term,

$$
M_{p}(r, X f) \lesssim \sup _{|z|<1 / 3}|X f(z)|+\int_{1 / 4}^{r} M_{p}(t, X \mathcal{R} f) d t
$$

By the harmonicity of $X R f$ and by the Cauchy estimate of the harmonic functions, we have $\sup _{|z|<1 / 3}|X f(z)| \lesssim \sup _{|z|<1 / 2}|f(z)|$. If we apply the inequality (2.8) to the right side integrand with $X \mathcal{R} f$ instead of $X f$, we have

$$
\begin{aligned}
M_{p}(r, X f) & \lesssim \sup _{|z|<1 / 2}|f(z)|+\int_{0}^{r} \int_{0}^{t} M_{p}\left(s, X \mathcal{R}^{2} f\right) d s d t \\
& =\sup _{|z|<1 / 2}|f(z)|+\int_{0}^{r}(r-s) M_{p}\left(s, X \mathcal{R}^{2} f\right) d s .
\end{aligned}
$$

Repeating this $k$ times we get $(2.6)$.

Next, apply (2.6) with $X=T^{\nu}$ to get

$$
M_{p}\left(r, T^{\nu} f\right) \lesssim \sup _{|z|<1 / 2}|f(z)|+\int_{0}^{r}(r-s)^{k-1} M_{p}\left(s, T^{\nu} \mathcal{R}^{k} f\right) d s .
$$

Noting the identity

$$
-\sum_{i \neq j} \overline{\mathcal{T}}_{i j} \mathcal{T}_{i j} f(z)=2(n-1) \mathcal{R} f(z)
$$

valid for all holomorphic $f$ on $B$, it follows that

$$
\begin{aligned}
M_{p}\left(r, T^{\nu} f\right) & \lesssim \sup _{|z|<1 / 2}|f(z)|+\sum_{|\mu|=|\nu|+2 k} \int_{0}^{r}(r-s)^{k-1} M_{p}\left(s, T^{\mu} f\right) d s \\
& =\sup _{|z|<1 / 2}|f(z)|+\sum_{|\mu|=|\nu|+2 k} r^{k} \int_{0}^{1}(1-t)^{k-1} M_{p}\left(t, T^{\mu} f_{r}\right) d t
\end{aligned}
$$

where $f_{r}(z)=f(r z)$. Applying Corollary 2.3 to the last integrand, we obtain

$$
M_{p}\left(r, T^{\nu} f\right) \lesssim \sup _{|z|<1 / 2}|f(z)|+\sum_{|\mu|=|\nu|+k} r^{k} \int_{0}^{1}(1-t)^{k / 2-1} M_{p}\left(\tilde{t}, T^{\mu} f_{r}\right) d t .
$$

Now the change of the variables $\tilde{t}=t+(1-t) / 4 \rightarrow s / r$ proves $(2.7)$.

Corollary 2.5. Let $X$ be the differential operators of the form (2.2) with weight $m$. If $k>m$, then for $1 / 2<r<1$ we have

$$
M_{p}(r, X f) \lesssim \sup _{|z|<1 / 2}|f(z)|+M_{p}\left(r, \mathcal{R}^{k} f\right) .
$$

Proof. Let $f_{r}(z)=f(r z)$. By (2.6) and Corollary 2.3 with the increasing property of $M_{p}\left(t, \mathcal{R} f_{r}\right)$, we have

$$
M_{p}(r, X f) \lesssim \sup _{|z|<1 / 2}|f(z)|+\int_{0}^{r}(r-t)^{k-1} M_{p}\left(t, X \mathcal{R}^{k} f\right) d t
$$


HOLOMORPHIC MEAN LIPSCHITZ FUNCTIONS ON THE UNIT BALL OF $\mathbb{C}^{n} 195$

$$
\begin{aligned}
& =\sup _{|z|<1 / 2}|f(z)|+r^{k} \int_{0}^{1}(1-t)^{k-1} M_{p}\left(t, X \mathcal{R}^{k} f_{r}\right) d t \\
& \lesssim \sup _{|z|<1 / 2}|f(z)|+\int_{0}^{1}(1-t)^{k-m-1} M_{p}\left(\tilde{t}, \mathcal{R}^{k} f_{r}\right) d t \\
& \lesssim \sup _{|z|<1 / 2}|f(z)|+M_{p}\left(r, \mathcal{R}^{k} f\right) .
\end{aligned}
$$

Lemma 2.6 (Hardy's inequalities [12]). Let $h$ be a non-negative function and $r>0$. Then
(i) $\left\{\int_{0}^{1}\left(\int_{0}^{x} h(y) d y\right)^{p} x^{-r-1} d x\right\}^{1 / p} \leq \frac{p}{r}\left(\int_{0}^{1}(y h(y))^{p} y^{-r-1} d y\right)^{1 / p}$;
(ii) $\left\{\int_{0}^{1}\left(\int_{x}^{1} h(y) d y\right)^{p} x^{r-1} d x\right\}^{1 / p} \leq \frac{p}{r}\left(\int_{0}^{1}(y h(y))^{p} y^{r-1} d y\right)^{1 / p}$.

Corollary 2.7. Let $a, b>0$. Then for non-negative increasing function $F$

$$
\int_{0}^{1}(1-r)^{a-1}\left(\int_{0}^{r}(r-t)^{b-1} F(t) d t\right)^{p} d r \lesssim \int_{0}^{1}(1-r)^{a+b p-1} F(r)^{p} d r .
$$

Proof. By a change of variables,

$$
\begin{aligned}
& \int_{0}^{1}(1-r)^{a-1}\left(\int_{0}^{r}(r-t)^{b-1} F(t) d t\right)^{p} d r \\
= & \int_{0}^{1} x^{a-1}\left(\int_{0}^{1-x}(1-x-t)^{b-1} F(t) d t\right)^{p} d x \\
= & \int_{0}^{1} x^{a-1}\left(\int_{x}^{1}(y-x)^{b-1} F(1-y) d y\right)^{p} d x .
\end{aligned}
$$

For the inner integral on the right side of (2.10), by setting $\delta(x)=\min \{2 x, 1\}$ and by using the increasing property of $F$, we have

$$
\begin{aligned}
& \int_{x}^{1}(y-x)^{b-1} F(1-y) d y \\
= & \int_{x}^{\delta(x)}(y-x)^{b-1} F(1-y) d y+\int_{\delta(x)}^{1}(y-x)^{b-1} F(1-y) d y \\
\leq & \frac{1}{b} x^{b} F(1-x)+2 \int_{\delta(x)}^{1} y^{b-1} F(1-y) d y \\
\leq & \frac{1}{b} x^{b} F(1-x)+2 \int_{x}^{1} y^{b-1} F(1-y) d y,
\end{aligned}
$$

where we used $(y-x)^{b} \leq y^{b}$ and $(y-x)^{-1} \leq 2 / y$ in the first inequality. Therefore, joining (2.10) and (2.11) we have

$$
\int_{0}^{1}(1-r)^{a-1}\left(\int_{0}^{r}(r-t)^{b-1} F(t) d t\right)^{p} d r
$$




$$
\lesssim \int_{0}^{1} x^{a-1+b p} F(1-x)^{p} d x+\int_{0}^{1} x^{a-1}\left(\int_{x}^{1} y^{b-1} F(1-y) d y\right)^{p} d x
$$

An application of Lemma 2.6 and a change of variables complete the proof.

We now are ready to prove Theorem 2.1.

Proof of Theorem 2.1. We prove (i) $\Longleftrightarrow$ (ii), (iii) $\Longleftrightarrow$ (iv), (ii) $\Longrightarrow$ (iii) \& (v), (iv) $\Longrightarrow$ (i) and (v) $\Longrightarrow$ (i).

(i) $\Longleftrightarrow$ (ii): It suffices to show (i) $\Longrightarrow$ (ii) since (ii) $\Longrightarrow$ (i) is trivial. Let $k>\alpha$ be fixed. We show $L_{l-\alpha}^{p, q}\left(\mathcal{R}^{l} f\right) \lesssim\|f\|_{H^{p}}+L_{k-\alpha}^{p, q}\left(\mathcal{R}^{k} f\right)$ for all integer $l>\alpha$. The case $l=k$ is obvious. If $l<k$, then by (2.6) of Lemma 2.4 and Corollary 2.7

$$
\begin{aligned}
& L_{l-\alpha}^{p, q}\left(\mathcal{R}^{l} f\right) \\
= & \left(\int_{0}^{1}(1-r)^{(l-\alpha) q-1} M_{p}\left(r, \mathcal{R}^{l} f\right)^{q} d r\right)^{1 / q} \\
\lesssim & \sup _{|z|<1 / 2}|f(z)|+\left\{\int_{0}^{1}(1-r)^{(l-\alpha) q-1}\left(\int_{0}^{r}(r-t)^{k-l-1} M_{p}\left(t, \mathcal{R}^{k} f\right) d t\right)^{q} d r\right\}^{1 / q} \\
\lesssim & \sup _{|z|<1 / 2}|f(z)|+\left(\int_{0}^{1}(1-r)^{(k-\alpha) q-1} M_{p}\left(r, \mathcal{R}^{k} f\right)^{q} d r\right)^{1 / q} \\
\lesssim & || f \|_{H^{p}}+L_{k-\alpha}^{p, q}\left(\mathcal{R}^{k} f\right) .
\end{aligned}
$$

If $l>k$, then by Corollary 2.3 with $X=\mathcal{R}^{l-k}$ together with the change of variables $\tilde{r} \rightarrow t$, it is easy to see that

$$
L_{l-\alpha}^{p, q}\left(\mathcal{R}^{l} f\right)=L_{l-\alpha}^{p, q}\left(\mathcal{R}^{l-k} \mathcal{R}^{k} f\right) \lesssim\|f\|_{H^{p}}+L_{k-\alpha}^{p, q}\left(\mathcal{R}^{k} f\right) .
$$

(iii) $\Longleftrightarrow$ (iv): The proof is analogous to the preceding one.

(ii) $\Longrightarrow$ (iii) \& (v): Let $|\nu|=k>2 \alpha$ and $l$ be another positive integer greater than $\alpha$. Then by (2.6) of Lemma 2.4

$$
\begin{aligned}
& \left(\int_{0}^{1}(1-r)^{(k / 2-\alpha) q-1} M_{p}^{q}\left(r, T^{\nu} f\right) d r\right)^{1 / q} \\
\lesssim & \sup _{|z|<1 / 2}|f(z)| \\
& +\left\{\int_{0}^{1}(1-r)^{(k / 2-\alpha) q-1}\left(\int_{0}^{r}(r-t)^{l-1} M_{p}\left(t, T^{\nu} \mathcal{R}^{l} f\right) d t\right)^{q} d r\right\}^{1 / q} .
\end{aligned}
$$

By Corollary 2.7, the last integral is bounded by

$$
\left(\int_{0}^{1}(1-r)^{(k / 2+l-\alpha) q-1} M_{p}^{q}\left(r, T^{\nu} \mathcal{R}^{l} f\right) d r\right)^{1 / q} .
$$


HOLOMORPHIC MEAN LIPSCHITZ FUNCTIONS ON THE UNIT BALL OF $\mathbb{C}^{n} 197$

Thus, by Corollary 2.3 with $X=T^{\nu}$ together with the change of variables $\tilde{r} \rightarrow t$, we have

$$
\begin{aligned}
& \left(\int_{0}^{1}(1-r)^{(k / 2-\alpha) q-1} M_{p}^{q}\left(r, T^{\nu} f\right) d r\right)^{1 / q} \\
\lesssim & \|f\|_{H^{p}}+\left(\int_{0}^{1}(1-r)^{(l-\alpha) q-1} M_{p}^{q}\left(r, \mathcal{R}^{l} f\right) d r\right)^{1 / q} .
\end{aligned}
$$

(iv) $\Longrightarrow$ (i): Let $k>\alpha$ and let $l>2 \alpha$. Applying (2.7) of Lemma 2.4 together with $(2.9)$

$$
\begin{aligned}
M_{p}\left(r, \mathcal{R}^{k} f\right) & \lesssim \sup _{|z|<1 / 2}|f(z)|+\sum_{|\mu|=l} \int_{0}^{r}(r-t)^{l / 2-1} M_{p}\left(t, T^{\mu} \mathcal{R}^{k} f\right) d t \\
& \lesssim \sup _{|z|<1 / 2}|f(z)|+\sum_{|\nu|=l+2 k} \int_{0}^{r}(r-t)^{l / 2-1} M_{p}\left(t, T^{\nu} f\right) d t .
\end{aligned}
$$

Then, as in the previous cases, using Corollary 2.7 we have

$$
L_{k-\alpha}^{p, q}\left(\mathcal{R}^{k} f\right) \lesssim\|f\|_{H^{p}}+\sum_{|\nu|=l+2 k} L_{k-\alpha+l / 2}^{p, q}\left(T^{\nu} f\right) \approx\|f\|_{H^{p}}+\sum_{|\nu|=l} L_{-\alpha+l / 2}^{p, q}\left(T^{\nu} f\right) .
$$

(v) $\Longrightarrow(\mathrm{i})$ : Take $k=l=1$ in the proof of (iv) $\Longrightarrow$ (i).

\section{A priori estimates}

In this section, we prepare connections between the $L^{p}$-modulus of continuity and the mean values of the radial derivatives (see [9] and [10] for the one variable case). Recall

$$
\omega_{p}(t, f)=\sup _{\|U-I\| \leq t}\left(\int_{\zeta \in S}|f(U \zeta)-f(\zeta)|^{p} d \sigma(\zeta)\right)^{1 / p}
$$

Theorem 3.1. Let $0<r<1$ and $f \in H^{p}(B)$. Then, we have

$$
M_{p}(r, \mathcal{R} f) \lesssim \int_{0}^{2 \pi} \frac{\omega_{p}(t, f)}{\left|1-r e^{-i t}\right|^{2}} d t
$$

Proof. For a fixed $z \in B$, let $f_{z}(\lambda)=f(\lambda z)$ where $\lambda \in \mathbb{D}$. Since $f_{z} \in H^{1}(\mathbb{D})$ every $z \in B$, it follows by one variable Cauchy integral representation that

$$
|\mathcal{R} f(r z)| \leq\left|f_{z}^{\prime}(r)\right| \lesssim \int_{0}^{2 \pi} \frac{\left|f\left(U_{t} z\right)-f(z)\right|}{\left|e^{i t}-r\right|^{2}} d t
$$

where $U_{t}$ is the unitary transformation defined by $U_{t} z=e^{i t} z=\left(e^{i t} z_{1}, e^{i t} z_{2}, \ldots\right.$, $\left.e^{i t} z_{n}\right)$. Using the representation $z=s \zeta$ with $\zeta \in S, 0<s<1$, we then have

$$
M_{p}(r s, \mathcal{R} f) \lesssim\left(\int_{S}\left(\int_{0}^{2 \pi} \frac{\left|f \circ U_{t}(s \zeta)-f(s \zeta)\right|}{\left|1-r e^{-i t}\right|^{2}} d t\right)^{p} d \sigma(\zeta)\right)^{1 / p}
$$


Since $\int_{S}\left|f \circ U_{t}(s \zeta)-f(s \zeta)\right|^{p} d \sigma(\zeta)$ is an increasing function of $s$ for each fixed $t$, by Minkowski's inequality we have

$$
M_{p}(r s, \mathcal{R} f) \lesssim \int_{0}^{2 \pi} \frac{1}{\left|1-r e^{-i t}\right|^{2}}\left\|f \circ U_{t}-f\right\|_{L^{p}(S)} d t .
$$

On the other hand,

$$
\left\|f \circ U_{t}-f\right\|_{L^{p}(S)} \leq \sup _{\|U-I\| \leq t}\|f \circ U-f\|_{L^{p}(S)}=\omega_{p}(t, f)
$$

because $\left\|U_{t}-I\right\|=\sup _{\zeta \in S}\left|U_{t} \zeta-\zeta\right| \leq t$. Therefore, by taking $s \rightarrow 1$, we have the desired inequality.

Theorem 3.2. Let $0<t<1 / 2$ and $f \in H^{p}(B)$. Then, we have

$$
\omega_{p}\left(t, f_{1-t}\right) \lesssim t \sup _{|z|<1 / 2}|f(z)|+t M_{p}(1-t, \mathcal{R} f) .
$$

Proof. Let $r=1-t$ and let $U$ be a unitary operator of $\mathbb{C}^{n}$ such that $\|U-I\| \leq t$. Then, there is another unitary operator $V$ of $\mathbb{C}^{n}$ such that $V^{-1} U V=D$, where $D$ is the diagonal matrix consisting of eigenvalues of $U$. Since $D$ is a unitary diagonal matrix, there exist real numbers $h_{1}, \ldots, h_{n}$ whose modulus does not exceed $\pi$, such that $D \zeta=\left(e^{i h_{1}} \zeta_{1}, e^{i h_{2}} \zeta_{2}, \ldots, e^{i h_{n}} \zeta_{n}\right)$. Also, by the unitary invariance of $d \sigma$, we have

$\left\|f_{r} \circ U-f_{r}\right\|_{L^{p}(S)}=\left\|f_{r} \circ U \circ V-f_{r} \circ V\right\|_{L^{p}(S)}=\left\|f_{r} \circ V \circ D-f_{r} \circ V\right\|_{L^{p}(S)}$.

For notational convenience, let $F=f \circ V, D \zeta=\left(e^{i h_{1}} \zeta_{1}, e^{i h_{2}} \zeta_{2}, \ldots, e^{i h_{n}} \zeta_{n}\right)=$ $e^{i h} \zeta$ and $|h|=\left(\sum_{1}^{n}\left|h_{j}\right|^{2}\right)^{1 / 2}$. Then we have

$$
\begin{aligned}
\left|F_{r} \circ D(\zeta)-F_{r}(\zeta)\right| & =\left|F\left(r e^{i h} \zeta\right)-F(r \zeta)\right| \\
& =\left|\int_{0}^{1} \frac{d}{d t}\left(F\left(r e^{i t h} \zeta\right)\right) d t\right| \\
& =\left|\int_{0}^{1} \sum_{j=1}^{n} i h_{j} r e^{i t h_{j}} \zeta_{j} \frac{\partial F}{\partial z_{j}}\left(r e^{i t h} \zeta\right) d t\right| \\
& \lesssim|h| \int_{0}^{1}\left|\nabla F\left(r e^{i t h} \zeta\right)\right| d t .
\end{aligned}
$$

Applying Minkowski's inequality, we obtain

$$
\left\|F_{r} \circ D-F_{r}\right\|_{L^{p}(S)} \lesssim|h| M_{p}(r, \nabla F) .
$$

On the other hand, from the identity

$$
|z|^{2}|\nabla F(z)|^{2}=|\mathcal{R} F(z)|^{2}+\sum_{i<j}\left|\mathcal{T}_{i j} F(z)\right|^{2}
$$

(see [7] for example), it follows that

$$
M_{p}(r,|\nabla F|) \lesssim M_{p}(r, \mathcal{R} F)+\sum_{i<j} M_{p}\left(r, \mathcal{T}_{i j} F\right)
$$


HOLOMORPHIC MEAN LIPSCHITZ FUNCTIONS ON THE UNIT BALL OF $\mathbb{C}^{n} 199$ provided $r>1 / 2$. And Corollary 2.5 gives

$$
M_{p}\left(r, \mathcal{T}_{i j} F\right) \lesssim \sup _{|z|<1 / 2}|F(z)|+M_{p}(r, \mathcal{R} F)
$$

Gathering up, we thus have

$$
\left\|F_{r} \circ D-F_{r}\right\|_{L^{p}(S)} \lesssim|h| \sup _{|z|<1 / 2}|F(z)|+|h| M_{p}(r, \mathcal{R} F) .
$$

Since $\frac{1}{2}\left|h_{j}\right| \leq\left|e^{i h_{j}}-1\right| \leq|D \zeta-\zeta|$ for each $j=1, \ldots, n$ and

$$
\sup _{\zeta \in S}|D \zeta-\zeta|=\|D-I\|=\|V D-V\|=\|U V-V\|=\|U-I\|,
$$

we have $\frac{1}{2 n}|h| \leq\|U-I\|$. This implies $|h| \leq 2 n t$ since $\|U-I\| \leq t$. Therefore, we have

$$
\left\|F_{r} \circ D-F_{r}\right\|_{L^{p}(S)} \lesssim t \sup _{|z|<1 / 2}|F(z)|+t M_{p}(1-t, \mathcal{R} F) .
$$

Since $\mathcal{R} F(z)=(\mathcal{R} f)(V z)$, this completes the proof.

Theorem 3.3. Let $0<t<1 / 2$. Let $f \in H^{p}(B)$ and $f_{t}(\zeta)=f(t \zeta)$. Then, we have

$$
\omega_{p}(t, f) \lesssim t \sup _{|z|<1 / 2}|f(z)|+t M_{p}(1-t, \mathcal{R} f)+\int_{0}^{t} M_{p}(1-s, \mathcal{R} f) d s .
$$

Proof. Let $r=1-t$ and let $U$ be a unitary transformation of $\mathbb{C}^{n}$. Then, from the inequality

$$
|f(U \zeta)-f(\zeta)| \leq|f(U \zeta)-f(r U \zeta)|+\left|f_{r}(U \zeta)-f_{r}(\zeta)\right|+|f(r \zeta)-f(\zeta)|
$$

we obtain, via the unitary invariance of $d \sigma$, that

$$
\|f \circ U-f\|_{L^{p}(S)} \lesssim\left\|f-f_{r}\right\|_{L^{p}(S)}+\left\|f_{r} \circ U-f_{r}\right\|_{L^{p}(S)} .
$$

Therefore,

$$
\omega_{p}(t, f) \lesssim \omega_{p}\left(t, f_{1-t}\right)+\left(\int_{S}|f(\zeta)-f(r \zeta)|^{p} d \sigma(\zeta)\right)^{1 / p} .
$$

Thus, in view of Theorem 3.2, we need an upper bound estimate for

$$
\left(\int_{S}|f(\zeta)-f(r \zeta)|^{p} d \sigma(\zeta)\right)^{1 / p}
$$

Since

$$
f(\zeta)-f(r \zeta)=\int_{r}^{1} \frac{d}{d s} f(s \zeta) d s=\int_{r}^{1} \mathcal{R} f(s \zeta) \frac{d s}{s},
$$

by Minkowski's inequality, we get

$$
\left(\int_{S}|f(\zeta)-f(r \zeta)|^{p} d \sigma(\zeta)\right)^{1 / p} \lesssim\left(\int_{S}\left|\int_{r}^{1} \mathcal{R} f(s \zeta) \frac{d s}{s}\right|^{p} d \sigma(\zeta)\right)^{1 / p}
$$




$$
\begin{aligned}
& \lesssim \int_{r}^{1}\left(\int_{S}|\mathcal{R} f(s \zeta)|^{p} d \sigma(\zeta)\right)^{1 / p} d s \\
& \lesssim \int_{0}^{t} M_{p}(1-s, \mathcal{R} f) d s .
\end{aligned}
$$

This completes the proof.

\section{Proof of Theorem 1.1}

In this section, we compare the two quantities which define Besov space and mean Lipschitz space respectively. For $0<\alpha<1$, we define the mean Lipschitz semi-norm $\Omega_{\alpha}^{p, q}(f)$ by

$$
\Omega_{\alpha}^{p, q}(f)=\left(\int_{0}^{1} \omega_{p}(t, f)^{q} \frac{1}{t^{1+\alpha q}} d t\right)^{1 / q}
$$

Recall the definition of $L_{\alpha}^{p, q}(g)$ in (2.1). Theorem 4.1 stated below together with Theorem 2.1 proves Theorem 1.1.

Theorem 4.1. Let $0<\alpha<1$. Then, for $f \in H^{p}(B)$ the following are equivalent.

(i) $\Omega_{\alpha}^{p, q}(f)<\infty$.

(ii) $L_{1-\alpha}^{p, q}(\mathcal{R} f)<\infty$.

Furthermore, the left side quantities are equivalent up to addition by $\|f\|_{H^{p}(B)}$.

Proof. First suppose (ii). Cauchy estimates gives that $\sup _{|z|<1 / 2}|f(z)| \lesssim$ $\|f\|_{H^{p}(B)}$. Thus, by Theorem 3.3 and Theorem 3.2, we have

$$
\begin{aligned}
\Omega_{\alpha}^{p, q}(f) & =\left(\int_{0}^{1} \frac{1}{t^{1+\alpha q}}\left(\omega_{p}(t, f)\right)^{q} d t\right)^{1 / q} \\
& \lesssim\|f\|_{H^{p}(B)}+L_{1-\alpha}^{p, q}(\mathcal{R} f)+\left\{\int_{0}^{1} \frac{1}{t^{1+\alpha q}}\left(\int_{0}^{t} M_{p}(1-s, \mathcal{R} f) d s\right)^{q} d t\right\}^{1 / q} \\
& \lesssim\|f\|_{H^{p}(B)}+L_{1-\alpha}^{p, q}(\mathcal{R} f)+\left(\int_{0}^{1} t^{(1-\alpha) q} M_{p}^{q}(1-t, \mathcal{R} f) \frac{d t}{t}\right)^{1 / q} \\
& \approx\|f\|_{H^{p}(B)}+L_{1-\alpha}^{p, q}(\mathcal{R} f),
\end{aligned}
$$

where we used Hardy's inequality for the second inequality.

Conversely, assume (i). By Theorem 3.1,

$$
L_{1-\alpha}^{p, q}(\mathcal{R} f) \leq\left\{\int_{0}^{1}(1-r)^{(1-\alpha) q-1}\left(\int_{0}^{2 \pi} \frac{1}{\left|1-r e^{-i t}\right|^{2}} \omega_{p}(t, f) d t\right)^{q} d r\right\}^{1 / q} .
$$

Note that for $0<r, t<1$, we have

$$
2\left|1-r e^{i t}\right| \geq[(1-r)+r(1-\cos t)]+|r \sin t| \gtrsim(1-r)+|t| .
$$


Therefore,

$$
\begin{aligned}
L_{1-\alpha}^{p, q}(\mathcal{R} f) \lesssim\|f\|_{H^{p}(B)} & +\left\{\int_{0}^{1}(1-r)^{(1-\alpha) q-1}\left(\int_{0}^{1} \frac{\omega_{p}(t, f)}{(1-r)^{2}+t^{2}} d t\right)^{q} d r\right\}^{1 / q} \\
\lesssim\|f\|_{H^{p}(B)} & +\left\{\int_{0}^{1} x^{(1-\alpha) q-1}\left(\int_{0}^{x} \frac{\omega_{p}(t, f)}{x^{2}} d t\right)^{q} d x\right\}^{1 / q} \\
& +\left\{\int_{0}^{1} x^{(1-\alpha) q-1}\left(\int_{x}^{1} \frac{\omega_{p}(t, f)}{t^{2}} d t\right)^{q} d x\right\}^{1 / q} .
\end{aligned}
$$

By Hardy's inequalities (i) and (ii) of Lemma 2.6, we thus have

$$
L_{1-\alpha}^{p, q}(\mathcal{R} f) \lesssim\|f\|_{H^{p}(B)}+\Omega_{\alpha}^{p, q}(f) .
$$

\section{References}

[1] P. Ahern and J. Bruna, Maximal and area integral characterizations of Hardy-Sobolev spaces in the unit ball of $\mathbf{C}^{n}$, Rev. Mat. Iberoam. 4 (1988), no. 1, 123-153.

[2] P. Ahern and W. Cohn, Besov spaces, Sobolev spaces, and Cauchy integrals, Michigan Math. J. 39 (1992), no. 2, 239-261.

[3] N. Arcozzi, R. Rochberg, and E. Sawyer, Carleson measures and interpolating sequences for Besov spaces on complex balls, Mem. Amer. Math. Soc. 182 (2006), no. 859, 1-163.

[4] P. L. Duren, Theory of $H^{p}$ Spaces, Academic Press, New York, 1970.

[5] K. M. Dyakonov, Besov spaces and outer functions, Michigan Math. J. 45 (1998), no. $1,143-157$.

[6] G. H. Hardy and J. E. Littlewood, Some properties of fractional integrals II, Math. Z. 34 (1932), no. 1, 403-439.

[7] M. Jevtić and M. Pavlović, On M-harmonic Bloch space, Proc. Amer. Math. Soc. 123 (1995), no. 5, 1385-1392.

[8] H. T. Kaptanoğlu, Carleson measures for Besov spaces on the ball with applications, J. Funct. Anal. 250 (2007), no. 2, 483-520.

[9] M. Pavlović, Lipschitz spaces and spaces of harmonic functions in the unit disc, Michigan Math. J. 35 (1988), no. 2, 301-311.

[10] On the moduli of continuity of $H^{p}$ functions with $0<p<1$, Proc. Edinb. Math. Soc. (2) 35 (1992), no. 1, 89-100.

[11] Ch. Pommerenke, Boundary Behavior of Conformal Maps, Springer-Verlag, 1992.

[12] E. M. Stein, Singular Integrals and Differentiability Properties of functions, Princeton University Press, New Jersey, 1970.

ERN GUN KWON

Department of Mathematics Education

Andong National University

ANDONG 760-749, KoreA

E-mail address: egkwon@andong.ac.kr

Hong Rae Cho

Department of Mathematics

Pusan National University

Pusan 609-735, Korea

E-mail address: chohr@pusan.ac.kr 
HyungWoOn KoO

Department of Mathematics

Korea UnIVERsity

SEOUL 136-713, KorEA

E-mail address: koohw@korea.ac.kr 